\title{
Trayectorias de control y covariación de variables como expresión del cambio cognitivo en la solución de un problema*
}

Control Paths and Covariation of Variables as an Expresion of Cognitive Change in Solving a Problem

Recibido: 12 de julio de 2015 | Aceptado: 05 de noviembre de 2015

\author{
Hugo Alberto Escobar Melo** \\ ROCIO ABELlO CORREA \\ JoRge CASTAÑo GaRcía \\ Pontificia Universidad Javeriana, Colombia
}

doi : 10.11144/Javeriana.upsy15-1.tccv

Para citar este artículo: Escobar Melo, H. G., Abello Correa, R., \& Castaño García, J. (2016). Trayectorias de control y co-variación de variables como expresión del cambio cognitivo en la solución de un problema. Universitas Psychologica, 15(1), 281-302. http://dx.doi.org/10.11144/Javeriana.upsy15-1.tccv

* Artículo de investigación. Proyecto de investigación ID 003237, "Caracterización del cambio como novedad cognitiva en la solución de un problema físico-matemático de proporcionalidad en estudiantes de la Pontificia Universidad Javeriana". Financiado por la Vicerrectoría de Investigación.

** Correo electrónico: escobarh@javeriana.edu.co; abellor@javeriana.edu.co; castanoj@javeriana. edu.co

\section{RES U MEN}

Esta investigación caracteriza los trayectos y trayectorias que siguen 136 sujetos universitarios al solucionar un problema físico-matemático de proporcionalidad, planteado a través de un software interactivo, con el objetivo de analizar la variabilidad intra e intersujeto respecto del tipo de control de variables y covariación utilizadas. Se evidenciaron cinco tipos de control de variables (a: No Control, b: Control Simple de dos intentos, c: Control Simple mayor a dos intentos, d: Control con Alternancia y e: Cambio Controlado) y cuatro de covariación (f: No covariación, g: Covariación Creciente, h: Covariación Decreciente y i: Covariación Mixta). Se utilizó el método microgenético con medidas cualitativas y cuantitativas. Los diversos trayectos y trayectorias atestiguan el cambio cognitivo en la solución del problema. Se concluye que existe significativa variabilidad en los procedimientos utilizados por la población, al igual que diferencias significativas respecto de trayectorias con control o covariación consolidados, comparadas con las que no lo tuvieron, igualmente entre los que presentaron trayectos con intermitencia y sin intermitencia.

Palabras clave

cambio cognitivo; control y covariación de variables; solución de problemas;

trayectos y trayectorias; sujetos universitarios

\begin{abstract}
A B S T R A C T
This research characterizes paths and trajectories followed by 136 university subjects while solving a physical-mathematical problem of proportionality, proposed through an interactive software, in order to analyze the intra- and inter-subject variability, regarding the type of control variables and co-variation used. Five types of control variables (a: No Control, b: Simple Control, c: Simple Control greater than two attempts, d: Alternating Control and e: Controlled Change) and four of co-variation (f: No co-variation, g: Increasing co-variation, $\mathrm{h}$ : Decreasing co-variation and i: Mixed co-variation) were evidenced. The microgenetic method with qualitative and quantitative measures was used. The various paths and trajectories witness cognitive change in solving the problem. It is concluded that there is a significant variability in the procedures used by the population, as well as significant differences with respect to trajectories with consolidated control or co-variation compared to those that did not have it, also among those with paths with intermittence and without intermittence. Keywords

Cognitive Change; Control and Co-variation of Variables; Problem Solving; Paths and Trajectories; University Subjects
\end{abstract}




\section{Introducción}

El desarrollo cognitivo desde una perspectiva metodológica microgenética pone de relieve el cambio local en el marco de un proceso de evolución global (Puche-Navarro, 2008, citado por Vasco \& Henao, 2008), entendido este último como transformación abierta, dinámica y compleja (van Geert \& Steebeek, 2005).

En este marco de ideas, les ha interesado a los autores de este estudio preguntarse por las características que asumen los patrones de ejecución que se configuran en términos de trayectos y trayectorias a la hora de resolver un problema físico-matemático de proporcionalidad de movimiento uniforme rectilíneo, como una alternativa teórico-metodológica, para estudiar la novedad cognitiva local y su complejidad, enmarcada en una concepción global del desarrollo.

La pregunta central de investigación fue la siguiente: icómo caracterizar el cambio como novedad cognitiva en la solución de una tarea problema de movimiento lineal con velocidad constante en resolutores universitarios?

Las preguntas específicas se expresaron de la siguiente manera: ¿cuáles son y cómo se caracterizan los distintos patrones de ejecución de los resolutores al enfrentarse a la tarea-problema objeto de exploración?, iexiste variabilidad intra e intersujeto en los patrones de ejecución al resolver la tarea-problema objeto de exploración?

\section{Desarrollo y cambio cognitivo ${ }^{1}$}

De acuerdo con Vasco y Henao (2008) se define el desarrollo como "un proceso que implica cambio o variaciones en el tiempo y una multiplicidad o complejidad en el espacio" (p. 6); en la perspectiva de los autores citados, se afirma que esta aproximación comprensiva del desarrollo exige un acercamiento de inteligibilidad a las variaciones individuales en las trayectorias que cada sujeto vive, en términos de

1 Con la colaboración de Ana María Rodríguez y Ursula Hinostroza, Auxiliares de Investigación, Psicólogas, Pontificia Universidad Javeriana, Bogotá-Colombia lo que permanece y lo que cambia, que dé cuenta de la complejidad, la multicausalidad y los factores influyentes multi, micro, meso y macronivel, que inciden en el desarrollo. Tal postura es coherente con los planteamientos contemporáneos de van Geert \& Steebeek (2005) sobre el desarrollo visto desde los sistemas dinámicos, no lineales y complejos.

Teniendo en cuenta lo planteado, puede afirmarse que son diversos los niveles de resolución en los que pueden estudiarse los macro y microprocesos del desarrollo. De acuerdo con Vasco y Henao (2008), se plantea que una modalidad de cambio como proceso progresivo tendencialmente puede estudiarse por medio de un nivel de resolución bajo. Un cambio como proceso local, que puede ser progresivo y a la vez regresivo, requiere de un estudio en un nivel de resolución más fino. Para efectos de la investigación de base, el interés se centró en el microproceso en términos de una escala de exploración fina en la que se preguntó por el cambio como novedad cognitiva.

El cambio cognitivo, de acuerdo con Pozo (2009), exige distinguir al menos tres tipos de transformaciones que tienen que ver con la amplitud y profundidad del cambio: enriquecimiento que supone nueva información; ajuste que en cierto sentido modifica las estructuras cognitivas, pero sin producir un nuevo ordenamiento, y reestructuración que las reformatea completamente. Desde esta perspectiva, el cambio conceptual, en el sentido estricto del término, no constituye una acumulación de conocimientos: es ante todo reorganización que reformatea las estructuras cognitivas.

Posner y colaboradores (1982), citados por Pozo (2009), afirman que se requieren cuatro condiciones para que se dé un cambio cognitivo: la insatisfacción del sujeto con sus explicaciones; disponer de una nueva concepción, ya sea porque el sujeto la puede construir o porque está mediada por un proceso educativo; que ésta sea plausible y, por último, que resulte más potente en sus posibilidades explicativas. El cambio cognitivo exige, entonces, que el sujeto sienta insatisfacción para explicar el mundo desde sus referentes previos, además de vislumbrar en el proceso de construcción de nuevas 
ideas, una alternativa de comprensión diferente y más convincente.

El cambio cognitivo resulta ser uno de los ejes centrales a la hora de estudiar áreas como el desarrollo y el aprendizaje, es por esto que su estudio y cómo abordarlo ha sido un tema de gran interés (Bermejo, 2005). No obstante, la dificultad que se enfrenta al momento de estudiar el cambio en sí mismo y conocer sus causas y mecanismos ha llevado a la creación de modelos y enfoques que pretenden acercarse a la naturaleza de este fenómeno. Entre estos enfoques se encuentra el modelo de los sistemas dinámicos.

El campo del desarrollo psicológico, como cambio evolutivo, implica estados, acciones y algún tipo de mecanismo (Escobar, 2003). El estado final es bien conocido: cuando lo encontramos en términos de conductas complejas, definidas, eficientes, y no dudamos en afirmar que ha habido evolución. Cuando no se ha producido dicho desarrollo y el sujeto opera de forma aparentemente primitiva llegando a afirmarse que ha tenido lugar una regresión o una oscilación. Así, desarrollo se opone a regresión. Sin embargo, esta última bien puede ser de forma legítima una determinada "vección evolutiva". Tomados los asuntos de esta manera, se diferenciarán dos tipos de cambio evolutivo: el 'transformacional' y el 'variacional'. El primero se asemeja al que se ha descrito por medio de las metáforas conocidas, de la línea, la escalera o aún la espiral ascendente. Implica nuevas estructuras y una dirección con ciertas continuidades y discontinuidades. El segundo, el cambio variacional, se expresa en términos de modificaciones acerca del funcionamiento, con continuidades, reversibilidades y, en particular, mediante aprendizajes locales y específicos dentro de una estructura de conjunto (Overton, 1997).

Se debe acentuar que no se está hablando de cualquier cambio. Se trata del cambio que transforma e integra. Es decir, de un cambio que produce relaciones y conductas novedosas. Subestructuras en competencia con las totalidades y dirección explicable. En dicho proceso de transformación e integración se anclan dos elementos insospechados. La elección y la pérdida. La elección no necesariamente evoca algo de corte democrático y consciente. Puede ser forzada, como en la situación de haber llegado al borde del abismo, donde en vez de devolverse y extinguirse, no queda más que la alternativa elocuente y digna de saltar al vacío. La elección siempre implica una conditio sine qua non en términos de pérdida. Si se elige una dirección con posibles logros, accidentes y pérdidas, se empieza a transitar por algún camino que a su vez conducirá al sujeto por lugares insospechados, con posibilidades de éxitos y fracasos, pero al haber elegido siempre habrá una posible pérdida inevitable. El cambio evolutivo debe ser, en consecuencia, entendido como una epigénesis probabilística (Piaget, 1977). Muchas veces dichas probabilidades no están disponibles para ser un buen sujeto en evolución, depende, en ocasiones, de en manos de qué árbitro se esté. Se pierde y se gana, depende del aumento de las probabilidades y de las condiciones a favor o en contra.

El cambio evolutivo como epigénesis probabilística lleva a la existencia de niveles y clústeres. De esta manera, la representación del desarrollo no es posible a través de una figura sencilla, sino de cuatro dimensiones, incluida la temporal, con diversas direcciones que llevan a romper, en consecuencia, con la metáfora de la escalera que asciende hacia el infinito. Redes de varios niveles, telarañas encontradas, trayectos y trayectorias elegidas y puntos de inflexión. Un cuadro de cuestiones así no nos limita a ser eternos autómatas replicantes. De hecho tenemos desempeños explícitos e implícitos que hablan de ascensos y descensos, equilibrios puntuados con saltos exitosos o fracasos colosales; intercambios e innovaciones que conducen a la diversidad (Pinker, 2013).

Como en la física, nada se destruye, todo se transforma y algo queda invariante, hay un juego entre lo que permanece y lo que cambia. Aquí lo nuevo y lo viejo coexisten. Y en esa dialéctica se crean nuevas relaciones que se expresan en el cambio novedoso de la conducta, que se hace más explícita y, al mismo tiempo, encriptada frente a contenidos y representaciones a los que no se tiene acceso, lo que devela la presencia de campos conductuales complejos (Karmiloff-Smith, 1994). 
Como evidencia de lo anterior, existen diversos estudios e investigaciones, por ejemplo, relativos a la microgénesis y el cambio cognitivo en la adquisición del cardinal numérico en Bermejo (2005); los modelos Bayesianos y funcionamientos inferenciales complejos en Ossa y Puche-Navarro (2010); las matrices de transición y los patrones de variabilidad cognitiva en Ossa (2013); la comprensión de sistemas de engranajes y el cambio cognitivo en niños de cuatro años en NavarroRoldan (2008), todos ellos bajo un enfoque metodológico que acude al análisis microgenético para el estudio del cambio en situaciones de resolución de problemas específicos.

Para Siegler (1996) desde los años 70, los teóricos del desarrollo en su estudio acerca del cambio han enfatizado en dos aspectos: en primer lugar han resaltado la importancia del cambio como medio para comprender el desarrollo y el aprendizaje, y en segundo lugar han manifestado la dificultad de abordarlo, planteando que la comprensión del cambio cognitivo es limitada. Como respuesta a lo anterior, a través de los años han surgido modelos y métodos que han permitido un acercamiento a la comprensión de este proceso, permitiendo incrementar su estudio y comprensión.

Torbeyns, Arnaud, Lemaire y Verschaffel (2004) abordan el cambio cognitivo desde la perspectiva de Siegler (1996), al afirmar que:

[...] el desarrollo de estrategias cognitivas ha sido tradicionalmente descrito en términos de la metáfora de escalera. Esta metáfora representa al cambio cognitivo como una serie de ecuaciones 1:1 entre las edades y formas de pensar. Los niños de una determinada edad usan una estrategia particular para resolver una tarea cognitiva; el cambio cognitivo implica un período de transición relativamente breve, durante el cual múltiples estrategias entran en conflicto entre sí, dando lugar a la sustitución de la estrategia anteriormente utilizada por una cualitativamente mejor. ${ }^{2}$ (p. 188)

$2[\ldots]$ the development of cognitive strategies has traditionally been portrayed in terms of the staircase metaphor. This metaphor depicts cognitive change as a series of 1:1 equations between ages
Según lo anterior, Siegler (1994, citado por Ossa, 2013) considera que el cambio cognitivo puede ser evidenciado en la aparición de nuevas estrategias y que el "por qué" de la presencia de la variabilidad puede encontrarse en los propios procesos de este fenómeno. De aquí la importancia de estudiar el cambio a través de los diversos procesos que realiza el sujeto durante cada una de los transformaciones, tal como acontece en la presente investigación.

En respuesta a los modelos tradicionales de cambio cognitivo, Siegler (1996) propone su teoría de superposición de olas (overlapping waves theory) como una nueva perspectiva para la comprensión del desarrollo, la cual se encarga de explicar la variabilidad que existe en la resolución de problemas por parte de los niños. Según esta teoría, los sujetos tienen acceso a un amplio rango de estrategias para la resolución de problemas y pueden elegir qué estrategia utilizar dependiendo de la naturaleza del problema; dichas estrategias van desde las más básicas hasta las más avanzadas (Farrington, Vanuxem, \& Stiller, 2009).

Teniendo en cuenta lo que implica el cambio y entendiendo la solución de problemas como "la función psicológica superior, entrelazada con el pensamiento abstracto, el razonamiento y la memoria operativa, entre otros" (Montealegre, 2011, p. 120), la solución de problemas se ha utilizado como medio para estudiar el cambio cognitivo en una tarea específica, pues ésta implica el uso de una serie de procesos y estrategias heurísticas utilizadas para encontrar la solución.

Los estudios evolutivos se han centrado en estudiar lo estable y universal dentro de las pautas generadas por el cambio y no en identificar los factores o procesos generadores del cambio. Así pues, el objetivo central del que parte el enfoque de los sistemas dinámicos es reconocer y analizar la variabilidad que se presenta en el funcionamiento cognitivo y conductual del ser humano, la cual se caracteriza por ser compleja y no lineal. En este sentido, ésta

and ways of thinking. Children of a given age use a particular strategy to solve a cognitive task; cognitive change involves a relatively brief period of transition, during which multiple strategies conflict with each other, leading to the substitution of the previously used strategy for a qualitatively better one. 
perspectiva describe la naturaleza del desarrollo no en términos de reglas simples, sino en función de ecuaciones que predicen familias de curvas irregulares y diversas (Gutiérrez, García, \& Luque, 2002).

Para Ossa (2013), la variabilidad ha sido estudiada extensivamente y diversos autores han aportado a la definición desde distintos abordajes. Entre estos autores se encuentran Piaget (1979), Yan y Fischer (2002), van Geert y van Djik (2002), Puche-Navarro, Combariza y Ossa (2003), entre otros. Cada una de las definiciones de estos teóricos es distinta, aun así en términos generales, se puede decir que entienden la variabilidad como una característica propia del desarrollo y del funcionamiento cognitivo que se presenta en trayectorias con un flujo de cambio constante que refleja la dinámica no lineal.

La perspectiva de la solución de problemas ha ayudado a la comprensión del cambio con métodos específicos para su estudio, a lo que se suma el método microgenético como uno de los más significativos. Este análisis se centra en el estudio de cambios individuales, "de pequeña magnitud que se producen en los procesos psicológicos de interés durante una única sesión de observación o experimental. El objetivo es poder estudiar el cambio mientras éste se produce" (Gutiérrez \& Carriedo, 2002, p. 78). Dicho análisis se basa en tres premisas básicas: estudiar la dinámica de los procesos de cambio y no los resultados estáticos, explicar dichos procesos y no quedarse en la descripción de los mismos y sólo a través de esta aproximación se puede llegar a comprender cómo se han formado las funciones psíquicas superiores.

Al considerar al desarrollo como un fenómeno no lineal, irregular y variable, instalado en una perspectiva de los sistemas dinámicos, se está aceptando que el método para explorar estos fenómenos ha de ser uno que sea capaz de capturar y dar cuenta de los trayectos y trayectorias desiguales y dinámicas que se presentan.

\section{Objetivo}

Identificar y caracterizar la variabilidad intra e intersujeto en lo relativo a los patrones de ejecución en la resolución de la tarea-problema objeto de exploración.

\section{Método}

\section{Categorías}

- Dimensión Control de variables: se refiere a mantener constante el valor de una variable por dos o más intentos mientras se cambia el valor de la otra.

- Covariación: se refiere a la relación entre las variables velocidad y duración del evento para producir un efecto en la distancia recorrida.

- Patrones de ejecución: regularidad en las dimensiones control o covariación en una sucesión de intentos.

\section{Participantes}

La muestra de los participantes en la investigación de base, de tipo intencional no probabilística, estuvo conformada por 136 estudiantes universitarios, tanto mujeres como hombres, entre los 17 y 25 años de edad, distribuidos así: 42 de la Facultad de Ciencias, 35 de la Facultad de Artes y 59 de la Facultad de Psicología.

\section{Tipo de estudio}

El tipo de estudio es descriptivo bajo una aproximación metodológica de análisis microgenético que permitió tanto un análisis cualitativo como uno cuantitativo de los datos recogidos sobre la solución de la tarea-problema.

De acuerdo con Siegler y Crowley (1991), el análisis microgenético posibilita observar registrar y analizar directamente la novedad cognitiva y los mecanismos del cambio. Para lograr dicho propósito se ha de atender a dos características: primero, la observación del proceder del resolutor, la cual ha de hacerse desde el principio del cambio hasta que este adquiere estabilidad; segundo, se requiere de una alta densidad de las observaciones para acercarse comprensivamente al cambio cognitivo. 


\section{Instrumento}

La tarea-problema que se propuso resolver en este estudio es un típico problema de movimiento rectilíneo de velocidad constante. Específicamente consiste en determinar la velocidad constante que debe imprimirse a un móvil y el tiempo que debe durar el evento-movimiento para cubrir una distancia que previamente se fija sobre un segmento de recta. Tanto la velocidad y el tiempo son dados en valores "escalares" (es decir, se da el valor que indica su extensión sin ninguna unidad). El resolutor debe determinar la velocidad (V) y la duración del movimiento $(\mathrm{T})$ de un móvil para que éste recorra la distancia D que separa el punto de partida y el punto de llegada (la meta) con un error de más o menos el 2,5\% de la distancia D. Se pueden realizar hasta 15 intentos para ejecutar la tarea.

El problema a resolver fue diseñado en un software interactivo que combina diversos lenguajes expresivos, gráfico, numérico y escritural, captura los valores que el resolutor da a la variables $\mathrm{V}$ y $\mathrm{T}$ en los distintos intentos, retroalimenta de forma inmediata el procedimiento hecho y, una vez terminada la tarea, permite grabar las verbalizaciones de los sujetos sobre su proceder. Adicionalmente cuenta con una caja de herramientas que se pueden utilizar para ayudarse en la resolución de la tareaproblema, además de una pantalla donde pueden escribir o dibujar y una calculadora. El software

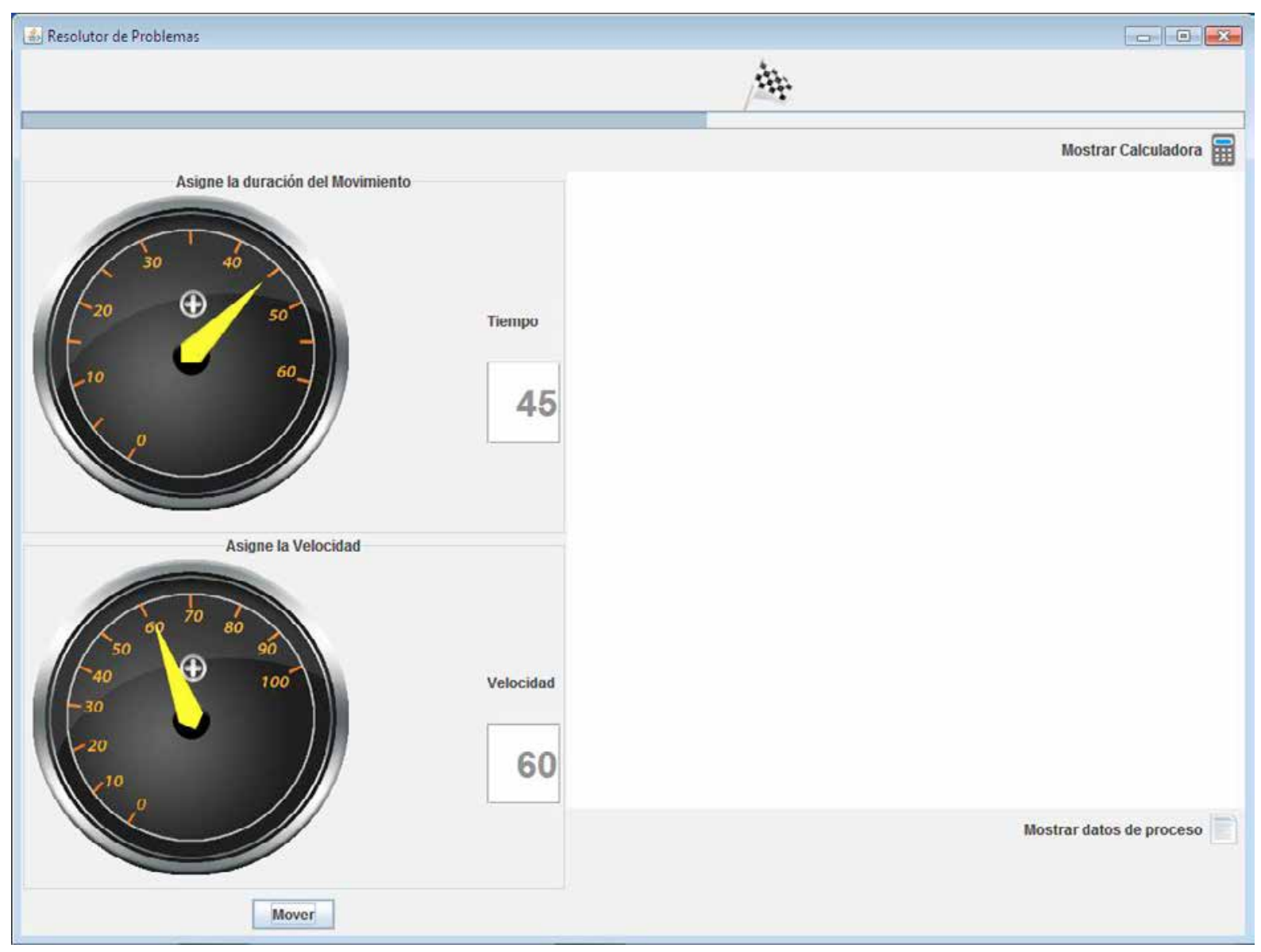

Figura 1. Interfaz del software URANUS

Fuente: elaboración propia 
URANUS analiza y grafica los procedimientos de los resolutores por segmentos para las dimensiones del control de variables y la covariación.

Dicho problema se presenta bajo una aplicación compilada y programada en lenguaje JAVA orientado a objetos. Posee un repositorio complejo de datos que permite seguir minuciosamente la actividad del sujeto y clasificar automáticamente.

\section{Metodología}

A partir del análisis microgenético utilizado, se identificaron los tipos de control de variables y covariación empleados por los sujetos en su proceder al resolver la tarea problema. A partir de dicha microgénesis, se caracterizaron los tipos de trayectos y trayectorias de los resolutores.

\section{Patrones de ejecución para la dimensión control}

$\boldsymbol{a}$ - No control: cuando de un intento al siguiente se cambian los valores de ambas variables sin que se aprecie que el resolutor haga algún tipo de control, en el sentido de dejar una de las variables de manera constante.

b - Control simple de dos intentos: cuando en una serie no superior a dos intentos el resolutor deja constante una de las dos variables y cambia el valor de la otra.

c - Control simple de más de dos intentos: cuando en una serie de tres o más intentos, el resolutor deja constante una de las dos variables y cambia el valor de la otra.

d - Control con alternancia: cuando en una serie de al menos tres intentos entre el primero y el segundo se hace un control simple y entre el segundo y el tercer intento se vuelve a hacer control simple, pero cambiando la variable que se había controlado, y así sucesivamente.

e - Cambio controlado de variables: cuando en una serie de dos intentos se cambian los valores de ambas variables en términos de incremento positivo o negativo no mayor a dos (2) unidades. Se aprecia que el resolutor asigna valores en el nuevo intento a partir del inmediatamente anterior para producir un cambio sutil en el desplazamiento del móvil. El criterio de un incremento positivo o negativo no mayor de dos (2) unidades se define con base en evidencias empíricas.

\section{Patrones de ejecución según la dimensión covariación}

f - No Covariación: se distinguen tres casos. No covariación 1: un segmento se califica como no covariación 1 cuando está compuesto por dos (2) intentos al mismo lado de la meta y el segundo intento queda más lejos de la meta que el anterior. No covariación 2: un segmento se califica como de no covariación 2, cuando está compuesto de tres intentos, los dos primeros están en lados opuestos de la meta y el tercero está más lejos de la meta que el primero. No covariación 3: un segmento se califica como de no covariación 3, cuando está compuesto de tres intentos, todos del mismo lado de la meta y el tercero está más lejos de la meta que el segundo. g - Covariación creciente: constituye una serie creciente de tres o más intentos donde en cada intento se acerca más a la meta que el anterior, incluso si en el último se pasa al otro lado de la meta. Puede haber un segmento de covariación creciente de dos (2) intentos cuando estos son los dos últimos que conducen a la meta.

$\boldsymbol{h}$ - Covariación decreciente: constituye una serie decreciente de tres o más intentos donde en cada intento se acerca más a la meta que el anterior, incluso si en el último se pasa al otro lado de la meta. Puede haber un segmento de covariación decreciente de dos (2) intentos cuando estos son los dos últimos que conducen a la meta.

$\boldsymbol{i}$ - Covariación mixta: un segmento se califica como de covariación mixta, cuando está compuesto de tres intentos, el primero y el segundo en lados opuestos de la meta, siendo el segundo un punto de inflexión ${ }^{3}$ y el tercero está más cerca de la meta que el primero. Caso especial: en presencia de trayectorias con cuatro intentos en las que los tres últimos configuran un segmento de covariación mixta y con los dos primeros no es posible calificarlos como f1;

3 Punto de inflexión: cuando de un intento a otro se cambia el sentido de las aproximaciones a la meta. 
los cuatro intentos se considerarán como un segmento único de covariación mixta.

El proceder del sujeto se segmenta según cada dimensión y de acuerdo con los criterios definidos. Se entiende entonces por segmento una sucesión de intentos que tiene un mismo patrón de ejecución. Los segmentos conforman trayectos que se configuran como parte de una trayectoria que muestra uno o más segmentos. Finalmente el proceder total del sujeto constituye una trayectoria que se refiere a toda la secuencia que sigue el sujeto.

\section{Procedimiento}

Se aplicó la tarea-problema bajo el software URANUS a la población objeto de estudio y, a partir de los resultados, se caracterizaron los trayectos y trayectorias según patrones de ejecución para las dimensiones de control y covariación con el fin de identificar la variabilidad intra e intersujeto.

\section{Resultados}

Como se planteó con anterioridad, la presente investigación se propuso identificar y caracterizar la variabilidad intra e intersujeto en lo relativo a los patrones de ejecución en la resolución de la tareaproblema objeto de exploración. Dicho objetivo se cumplió en tanto los resultados expresan que la variabilidad de los sujetos al utilizar por lo menos cinco formas de control de variables claramente identificadas, además de cuatro tipos de covariación. Dicha utilización no es uniforme, lo que enfatiza el cambio, ya que cada sujeto finalmente se caracteriza por una trayectoria definida que expresa su proceder. Se pueden igualmente encontrar pequeñas agrupaciones de sujetos que la comparten (véanse las Figuras 2 a 7).

Los sujetos pueden empezar con la utilización de algún tipo de control e ir cambiándolo a lo largo de la solución del problema. Es así que existen trayectorias sin control de variables (sin covariación), con control de variables (con covariación), y que éstas últimas pueden ser consolidadas o no consolidadas. Se observó igualmente que las trayectorias evidencian mejoramiento, es decir inician sin control o sin covariación y en algún momento pasan a segmentos que evidencian algún tipo de control o covariación y que éstas pueden ser con intermitencia, es decir, que tienen segmentos intermedios de no control (no covariación), además de trayectorias sin intermitencia, en las que se observa que efectivamente no existen segmentos intermedios de no control (no covariación, véase Tabla 1).

El análisis cualitativo permitió caracterizar una trayectoria como una secuencia de los patrones de ejecución, según control (a, b, c, d y e) o según covariación (f, $\mathbf{g}, \mathbf{h}, \mathbf{i})$ descritos en la metodología.

En la Tabla 1 se observa que el $33.1 \%$ de las trayectorias frente a la dimensión control de variables

TABLA 1.

Cambio en las trayectorias según la dimensión control

\begin{tabular}{|c|c|c|c|}
\hline \multicolumn{2}{|c|}{ TIPO TRAYECTORIAS } & $\begin{array}{l}\text { Número de } \\
\text { sujetos }\end{array}$ & $\%$ \\
\hline \multicolumn{2}{|c|}{ SIN CONTROL Empieza y termina sin control } & 6 & 4.4 \\
\hline \multirow{2}{*}{$\begin{array}{l}\text { CON CONTROL. Empieza y termina } \\
\text { con control }\end{array}$} & $\begin{array}{l}\text { NO CONSOLIDADO } \\
\text { (Hay segmentos intermedios de no control) }\end{array}$ & 14 & 10.3 \\
\hline & $\begin{array}{l}\text { CONSOLIDADO } \\
\text { (NO hay segmentos intermedios de no control) }\end{array}$ & 45 & 33.1 \\
\hline \multicolumn{2}{|c|}{ DESMEJORANTE. Empieza con control y termina sin control. } & 1 & 0.7 \\
\hline \multirow{2}{*}{$\begin{array}{l}\text { MEJORANTE Empieza sin control y } \\
\text { termina con control. }\end{array}$} & $\begin{array}{l}\text { CON INTERMITENCIA } \\
\text { (hay segmentos intermedios de no control) }\end{array}$ & 13 & 9.6 \\
\hline & $\begin{array}{l}\text { SIN INTERMITENCIA } \\
\text { (No hay segmentos intermedios de no control) }\end{array}$ & 57 & 41.9 \\
\hline
\end{tabular}

Fuente: elaboración propia 
TABLA 2.

Cambio en las trayectorias según la dimensión covariación

\begin{tabular}{|c|c|c|c|}
\hline \multicolumn{2}{|l|}{ TIPO TRAYECTORIAS } & $\begin{array}{l}\text { Número de } \\
\text { sujetos }\end{array}$ & $\%$ \\
\hline \multicolumn{2}{|c|}{ SIN COVARIACION Empieza y termina sin covariación } & 2 & 1.5 \\
\hline \multirow{2}{*}{$\begin{array}{l}\text { CON COVARIACION. Empieza y } \\
\text { termina con covariación }\end{array}$} & $\begin{array}{l}\text { NO CONSOLIDADA } \\
\text { (Hay segmentos intermedios de no covariación) }\end{array}$ & 12 & 8.8 \\
\hline & $\begin{array}{l}\text { CONSOLIDADA } \\
\text { (NO hay segmentos intermedios de no covaria- } \\
\text { ción) }\end{array}$ & 98 & 72.1 \\
\hline \multicolumn{2}{|c|}{ DESMEJORANTE. Empieza con covariación y termina sin covariación. } & 5 & 3.7 \\
\hline \multirow{2}{*}{$\begin{array}{l}\text { MEJORANTE Empieza sin covaria- } \\
\text { ción y termina covariación }\end{array}$} & $\begin{array}{l}\text { CON INTERMITENCIA } \\
\text { (hay segmentos intermedios de no covariacion) }\end{array}$ & 2 & 1.5 \\
\hline & $\begin{array}{l}\text { SIN INTERMITENCIA } \\
\text { (No hay segmentos intermedios de no covaria- } \\
\text { ción) }\end{array}$ & 17 & 12.5 \\
\hline
\end{tabular}

Fuente: elaboración propia

son de "control consolidado", mientras que en la dimensión covariación el 72.1\% son de "covariación consolidada" (Tabla 2). Dichas trayectorias indican que los sujetos inician y terminan la tarea con algún tipo de control diferente a "a" (no control) o con algún tipo de covariación diferente a "f" (no covariación) y que a lo largo de sus trayectorias no se encuentra ningún segmento sin control o sin covariación.

Aunque estas trayectorias no presentan cambios que van de control a no control o de covariación a no covariación o viceversa, alguna de ellas sí presentan cambios menos drásticos, es decir, de un tipo de control distinto de 'no control' (o de un tipo de covariación distinto de 'no covariación') a otro tipo de control distinto de 'no control' (o de covariación 'no covariación'), en otras palabras, de control a control o de covariación a covariación. Por ejemplo, una trayectoria como "bcb", que es de control consolidado, muestra dos cambios: pasa de controlar una de las variables en dos intentos a controlar la otra variable en más de dos intentos $(\mathbf{b}=>\mathbf{c})$ y después, nuevamente, pasa a controlar la primera variable en dos intentos $(\mathbf{c}=>\mathbf{b})$.

De esta manera se podría afirmar que un poco más de $2 / 3$ de las trayectorias presentan algún tipo de cambio cuando se estudia la dimensión control y un poco más de $1 / 4$ cuando se estudia la dimensión covariación. Estos datos ponen en evidencia el cambio y la variabilidad en la forma como los sujetos afrontan la tarea.

Las trayectorias de "control no consolidado" (10.3\%, Tabla 1) y las de " covariación no consolidada" (8.8\%, Tabla 2) aunque tampoco muestran cambios globales debido a que sólo comparan los segmentos primero y último, puede suceder que algunas de ellas tengan cambios en segmentos intermedios; si son trayectorias de más de dos segmentos puede suceder que en el paso de un segmento a otro se produzca un cambio drástico de control a no control ${ }^{4}$ o de covariación a no covariación, o viceversa, o no de control a control, o de no covariación a covariación.

Claramente las trayectorias mejorantes y desmejorantes indican estos cambios drásticos en alguna de las dos dimensiones. El 51.5\% de las trayectorias muestran estos tipos de cambios en la dimensión control (Tabla 1) y el 14\% en la dimensión covariación (Tabla 2).

4 Para simplificar, siempre que no sea indispensable se dirá no control para referirse a un segmento cuyo control es de tipo "a" y control para referirse a un segmento cuyo tipo es distinto de "a" (b, c, d o e). De forma semejante se hará con covariación: no covariación es "f" y covariación es distinta de "f" (g, h, e i). 
La lectura de la Tabla 3 requiere comprender la medida del "tamaño promedio de segmentos", resultado de dividir el número de intentos sobre el número de segmentos. Este valor constituye un indicador de la frecuencia del cambio. Se interpreta así: a menor valor hay menos intentos entre cambio y cambio, y a mayor valor, hay más intentos, por ejemplo, el valor 3.3 de las trayectorias sin control significa que en promedio cada 3.3 intentos se cambia de tipo de control.

En dicha tabla se puede observar que el tamaño promedio de los segmentos en las trayectorias mejorantes con intermitencia según la dimensión control es el de menor valor (1.6 intentos/segmentos).

Este dato puede interpretarse en términos de que aquellas trayectorias en las que el sujeto exhibe que no posee control al comienzo, pero intenta buscarlo y termina teniendo éxito, son precisamente aquellas en las que los cambios en los tipos control se dan con más frecuencia. Este hecho parece corroborarse con las trayectorias de control no consolidado (2 intentos/segmentos) que, como su nombre lo indica, son trayectorias en las que si bien el sujeto inicia la resolución de la tarea exhibiendo control, lo pierde unos pocos intentos después para volver a recuperarlo posteriormente. En contraste, las trayectorias que exhiben poca frecuencia de cambio son las trayectorias sin control (3.3 intentos/ segmentos) o con control consolidado (3.1 intentos/ segmentos).

De forma análoga sucede con la dimensión covariación, como se aprecia en la Tabla 3. Precisamente las trayectorias mejorantes con intermitencia (2.9 intentos/segmentos) y de covariación no consolidada (2.6 intentos/segmentos) son las que tienen menor valor en el tamaño promedio de los segmentos, medida indicadora de mayor frecuencia de cambio, en contraste con las trayectorias sin covariación (9 intentos/segmentos) y con covariación consolidada (3.8 intentos/segmentos) con menor frecuencia de cambio.

Al comparar las diferentes trayectorias por el promedio de intentos (Tabla 3), que es la medida de la menor o mayor eficiencia para resolver la tarea, se aprecia que las trayectorias de control consolidado (4.8 intentos, el menor valor) y de covariación consolidada (4.5 intentos, el menor valor) y las de control mejorante sin intermitencia (5.2 intentos, el tercer menor valor) y covariación mejorante sin intermitencia (7.2 intentos, el segundo menor valor), son las más eficientes. Mientras que la mejorantes con intermitencia (8.5 intentos según control, el mayor valor y 11.5 según covariación, el segundo mayor valor) son las menos eficientes.

TABLA 3.

Promedio de segmentos, de intentos y de tamaño promedio de segmentos según las dos dimensiones

\begin{tabular}{|c|c|c|c|c|c|c|c|}
\hline \multirow{2}{*}{\multicolumn{2}{|c|}{ TIPO TRAYECTORIAS }} & \multicolumn{3}{|c|}{ Control } & \multicolumn{3}{|c|}{ covariación } \\
\hline & & $\begin{array}{l}\text { Promedio } \\
\text { segmentos }\end{array}$ & $\begin{array}{l}\text { Promedio } \\
\text { intentos }\end{array}$ & $\begin{array}{c}\text { Tamaño } \\
\text { promedio } \\
\text { segmentosa }\end{array}$ & $\begin{array}{l}\text { Promedio } \\
\text { segmentos }\end{array}$ & $\begin{array}{l}\text { Promedio } \\
\text { intentos }\end{array}$ & $\begin{array}{l}\text { Tamaño } \\
\text { promedio } \\
\text { segmentos }\end{array}$ \\
\hline \multicolumn{2}{|c|}{$\begin{array}{l}\text { SIN CONTROL O SIN } \\
\text { COVARIACIÓN }\end{array}$} & 2.3 & 6.2 & 3.3 & 2.0 & 12.0 & 9.0 \\
\hline \multirow{2}{*}{$\begin{array}{l}\text { CON CONTROL } \\
\text { OCON } \\
\text { COVARIACIÓN }\end{array}$} & $\begin{array}{l}\text { NO } \\
\text { CONSOLIDADO }\end{array}$ & 4.4 & 8.5 & 2.0 & 3.2 & 8.4 & 2.6 \\
\hline & CONSOLIDADO & 1.8 & 4.8 & 3.1 & 1.3 & 4.5 & 3.8 \\
\hline \multicolumn{2}{|l|}{ DESMEJORANTE } & 2.0 & 5.0 & 2.0 & 2.0 & 7.8 & 3.9 \\
\hline \multirow{2}{*}{ MEJORANTE } & $\begin{array}{l}\text { CON } \\
\text { INTERMITENCIA }\end{array}$ & 5.3 & 8.5 & 1.6 & 4.0 & 11.5 & 2.9 \\
\hline & $\begin{array}{l}\text { SIN } \\
\text { INTERMITENCIA }\end{array}$ & 2.4 & 5.2 & 2.2 & 2.1 & 7.2 & 3.5 \\
\hline
\end{tabular}

${ }^{a}$ El tamaño promedio de segmentos se calcula primero para cada sujeto y después se hace el promedio para los sujetos del tipo de trayectoria correspondiente.

Fuente: elaboración propia 
La incidencia de las dos dimensiones en la menor o mayor eficiencia con la que se resuelve la tarea, se corrobora en la diferencia en el promedio de intentos entre la trayectoria que tiene algún segmento con no control o no covariación con las que en todos sus segmentos son de control y de covariación. Al revisar los datos se encuentra que, según la dimensión control, se identifican 91 trayectorias con algún segmento de no control (control tipo a) que utilizan en promedio 6.3 intentos, mientras hay 45 trayectorias con todos sus segmentos de control (control tipo $\mathbf{b}, \mathbf{c}, \mathrm{d}$, o e) que en promedio necesitan de 4.8 intentos. Según la covariación la situación es análoga, aunque en este caso la diferencia es aún más marcada: 38 trayectorias tienen algún segmento con no covariación y necesitan en promedio de 8.2 intentos y 49 de ningún segmento de no covariación que en promedio necesitan de 4.9 intentos. Aunque en esta muestra la dimensión covariación marca mayor diferencia que la dimensión control entre las trayectorias que siempre manejan covariación o control de aquellas que al menos en un segmento no lo hacen, no se puede afirmar que el sujeto que está en posición de la dimensión covariación, pero no de control, resuelve la tarea de forma más eficiente que el que no maneja covariación y sí control. Antes de hacer alguna afirmación al respecto, conviene ana- lizar las trayectorias de manera simultánea según las dos dimensiones.

En la Tabla 4 se encuentra una única trayectoria que a la vez presenta no control y no covariación. Esta trayectoria agota los 15 intentos que se daban para intentar resolver la tarea. Mientras que se encuentran 51 trayectorias que presentan control y covariación, bien sea consolidado o mejorantes sin intermitencia (40 de control y covariación consolidada y 11 de control y covariación, mejorante sin intermitencia), el promedio de intentos es 5.2; estos datos muestran una clara diferencia en la eficiencia con la que se resuelve la tarea cuando no se manejan ambas dimensiones que cuando se manejan.

Se efectuaron medidas de ANOVA con prueba post hoc HSD Tukey para comprobar si existían diferencias significativas entre los diversos tipos de trayectorias para atestiguar con solidez y significancia la evidencia de la variabilidad. Ya se había encontrado indicios de ello (véanse las Tablas $1 \mathrm{a}$ 3) y así lo evidencian los indicadores del promedio de segmentos, el promedio de intentos de las trayectorias y el tamaño promedio de los segmentos.

El promedio de segmentos expresa la mayor o menor homogeneidad de las trayectorias, en tanto que ofrece una medida del número de cambios que se identifican a lo largo de la trayectoria. El prome-

\section{TABLA 4.}

Tabla de contingencia tipo de trayectoria según control * tipo de trayectoria según covariación

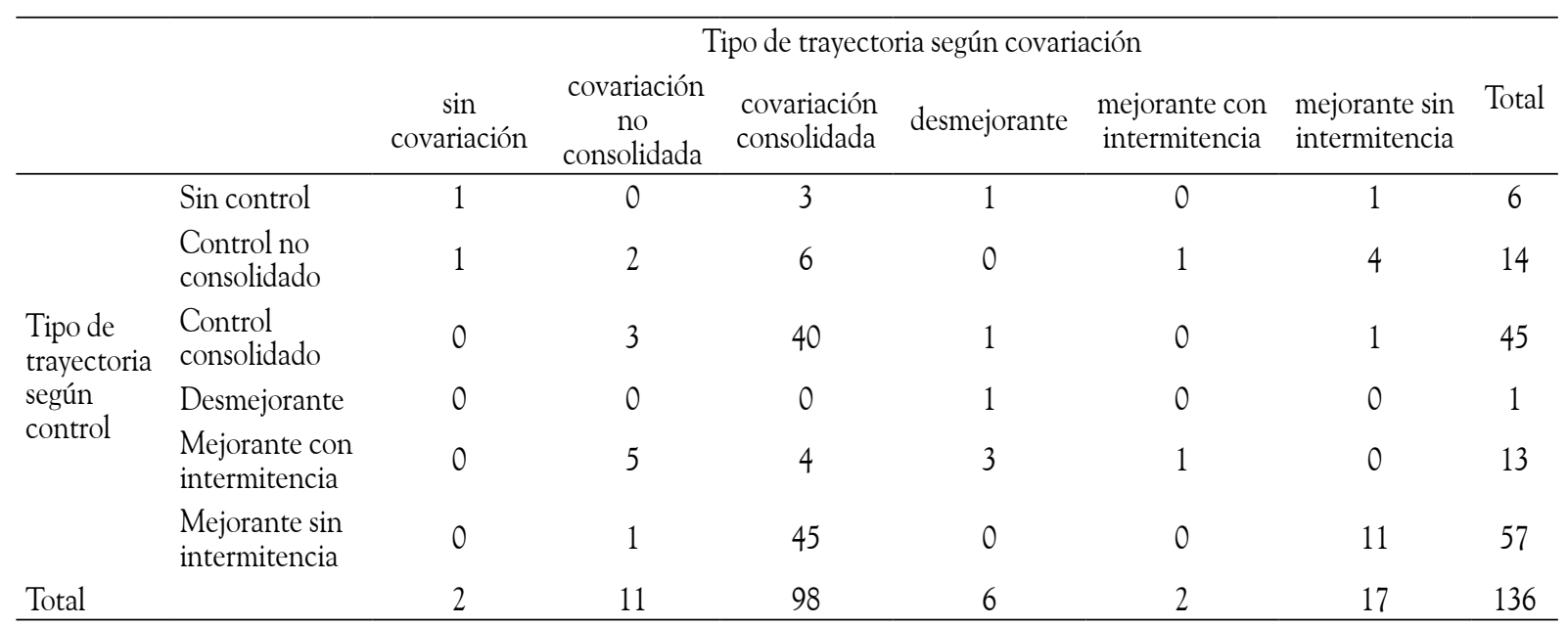

Fuente: elaboración propia 
dio de intentos de la trayectoria da cuenta de la eficacia, es decir, qué tantos intentos fueron necesarios para resolver la tarea. El promedio de intentos por segmento da cuenta del tamaño promedio de segmentos, denota la rapidez del cambio y que puede tomarse como otra medida de la variabilidad.

Tanto la homogeneidad y el tamaño promedio de los segmentos ofrecen una idea de la variabilidad y la rapidez con la que se da el cambio; en este contexto, una hipótesis plausible puede ser que los sujetos que siguen trayectorias, según las dimensiones control y covariación, de tipo consolidado o mejorante sin intermitencia, tenderían a ser más homogéneas (es decir, un menor valor en el número de segmentos) y a ser menos variables (es decir, un mayor valor en el promedio de intentos por seg- mento), mientras que las trayectorias de control no consolidado o de covariación no consolidada son menos homogéneas (el número de segmentos de las trayectorias es mayor) y tienden a tener mayor variabilidad, es decir, a tener un valor menor en el promedio de intentos por segmentos.

La Figura 2 evidencia que los sujetos con control consolidado y mejorante sin intermitencia se diferencian significativamente de los demás. En este sentido, presentan un promedio menor en el número de segmentos respecto de los demás grupos (control no consolidado y mejorante con intermitencia), lo que va en la línea de la hipótesis planteada con anterioridad.

La Figura 3 evidencia diferencias significativas entre los grupos con control consolidado y mejo-

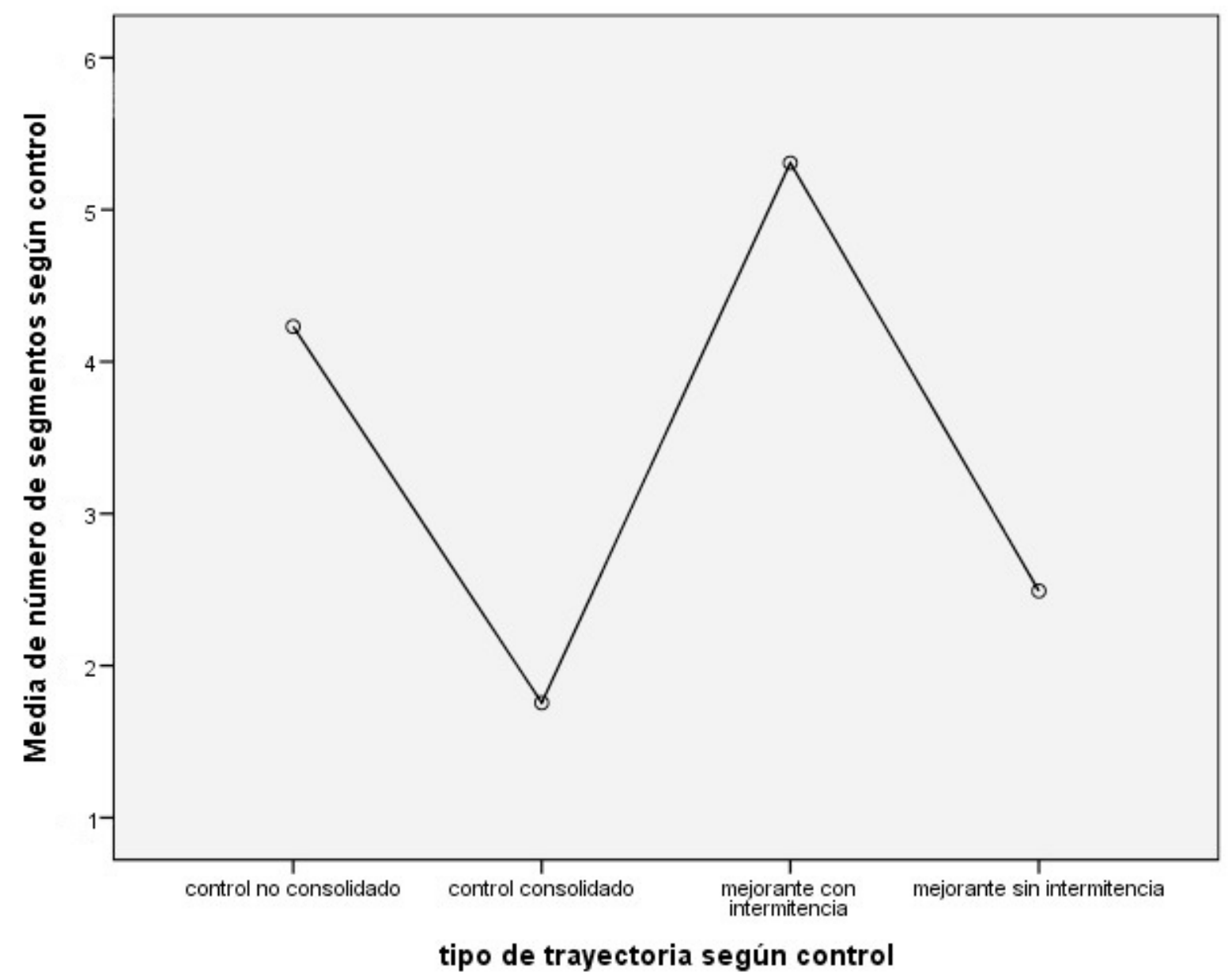

Figura 2. Promedio segmentos según control de variables

Fuente: elaboración propia 


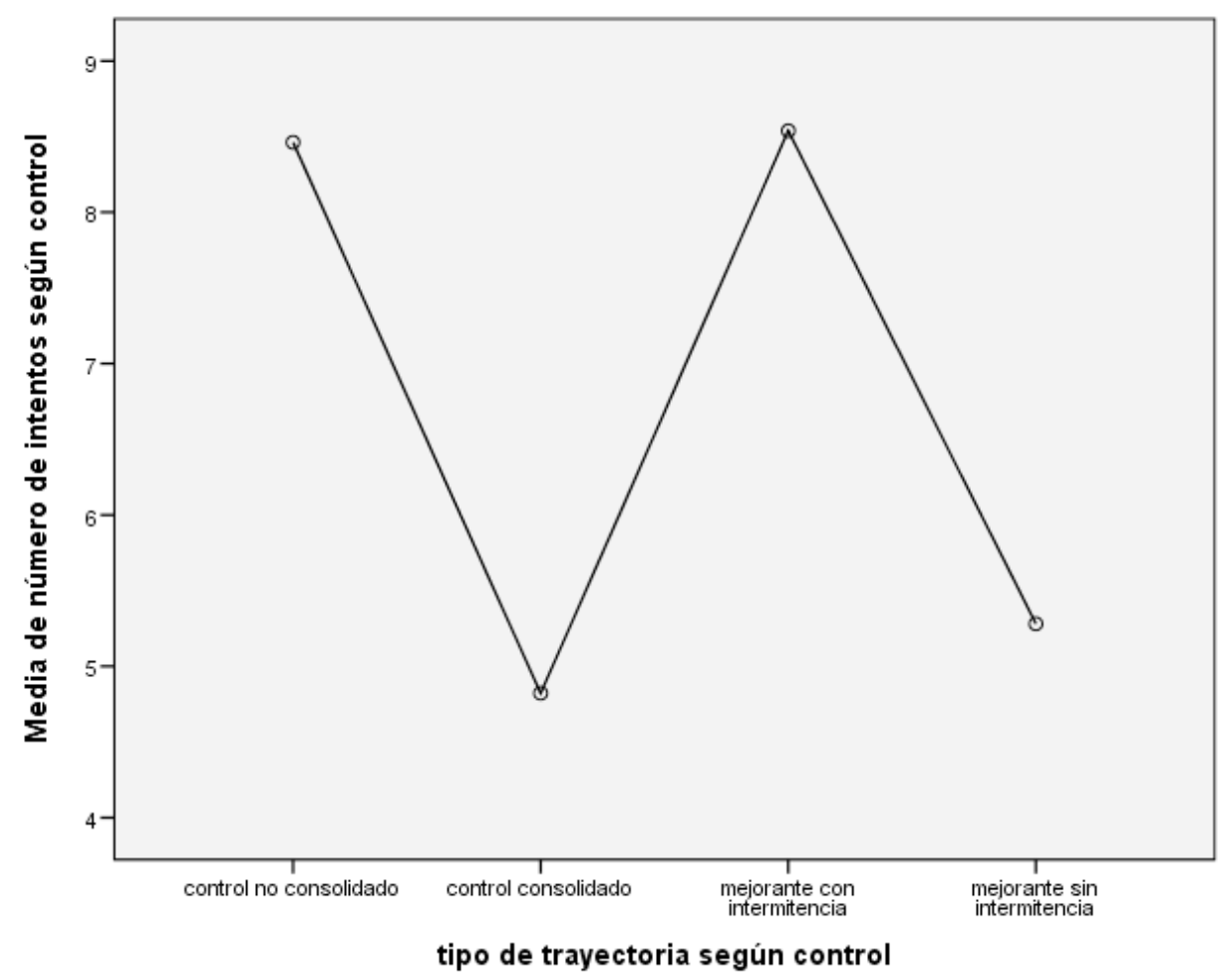

Figura 3. Número intentos por trayectoria según control de variables

Fuente: elaboración propia

rantes sin intermitencia, en contraste con los que no poseen control de variables o son mejorantes con intermitencia respecto del promedio de intentos; la mayor tasa de intentos se observa en estos últimos y corresponde a lo que se ha planteado en términos de eficiencia en los grupos que aún no utilizan plenamente el control de variables. Esta Figura igualmente permite observar que el grupo de sujetos con control consolidado de variables muestra un menor número de intentos al interior de sus segmentos, probablemente porque una solución eficiente del problema implica, por regla, un menor número de segmentos.

La Figura 4 expresa que los sujetos con control consolidado se diferencian de los demás grupos respecto del tamaño promedio de los segmentos. En esta Figura se evidencia que el grupo con control consolidado es significativamente diferente a todos los demás. Los sujetos pertenecientes a este grupo exhiben un tamaño de segmento más grande; para los sujetos con control no consolidado y mejorantes con intermitencia el tamaño promedio de sus segmentos es menor; lo anterior argumenta que los primeros son consistentes en la aplicación de alguna forma de control; cabe anotar que no se encontraron diferencias significativas de esta medida en la dimensión de covariación.

La Figura 5 muestra una diferencia significativa entre los grupos con covariación consolidada y mejorante sin intermitencia, frente a las de covariación no consolidada y mejorante con intermitencia, en términos del promedio de segmentos utilizados. Igualmente se observa el contraste entre el grupo con covariación consolidada y el grupo mejorante con intermitencia; este último utiliza más segmentos en el intento de resolver el problema con una conducta variacional. En este caso, los primeros grupos muestran un promedio menor de segmentos, como en el caso anterior, frente a la 


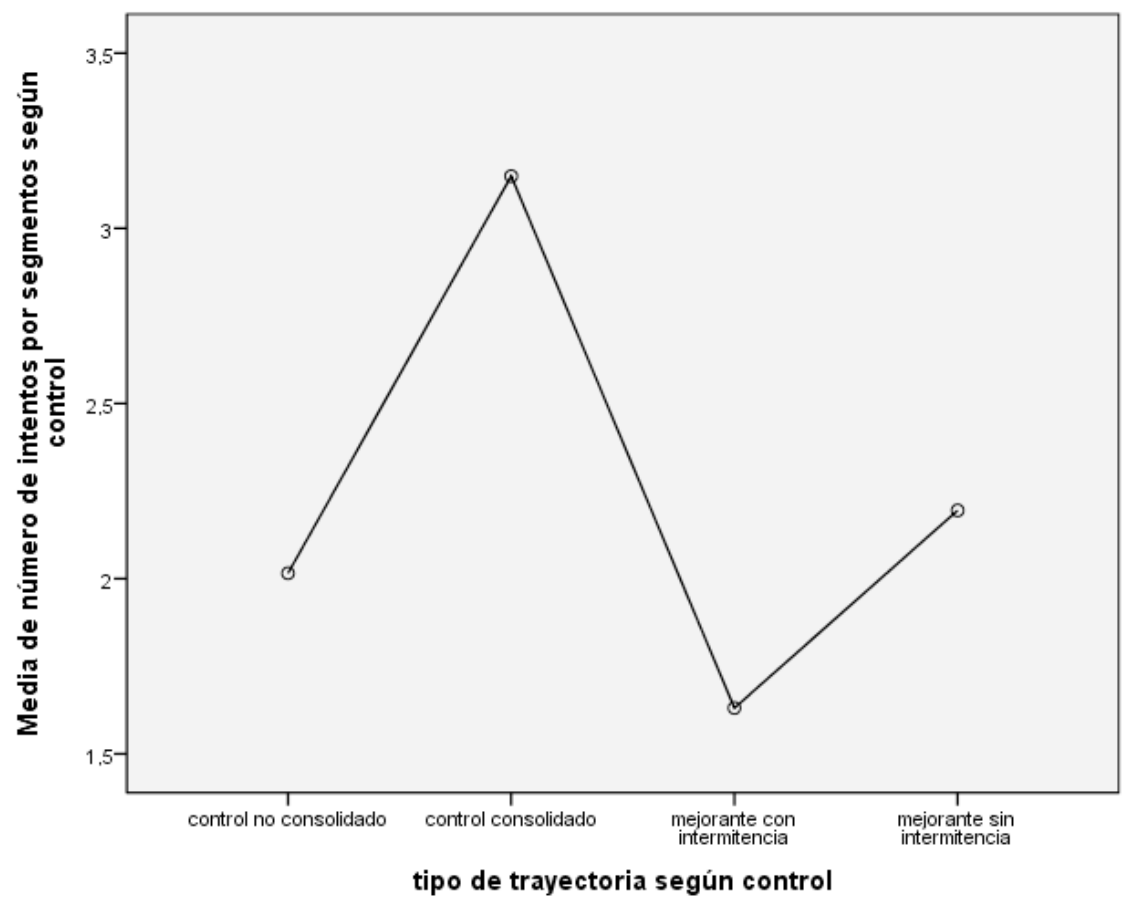

Figura 4. Tamaño promedio de segmentos según control de variables

Fuente: elaboración propia

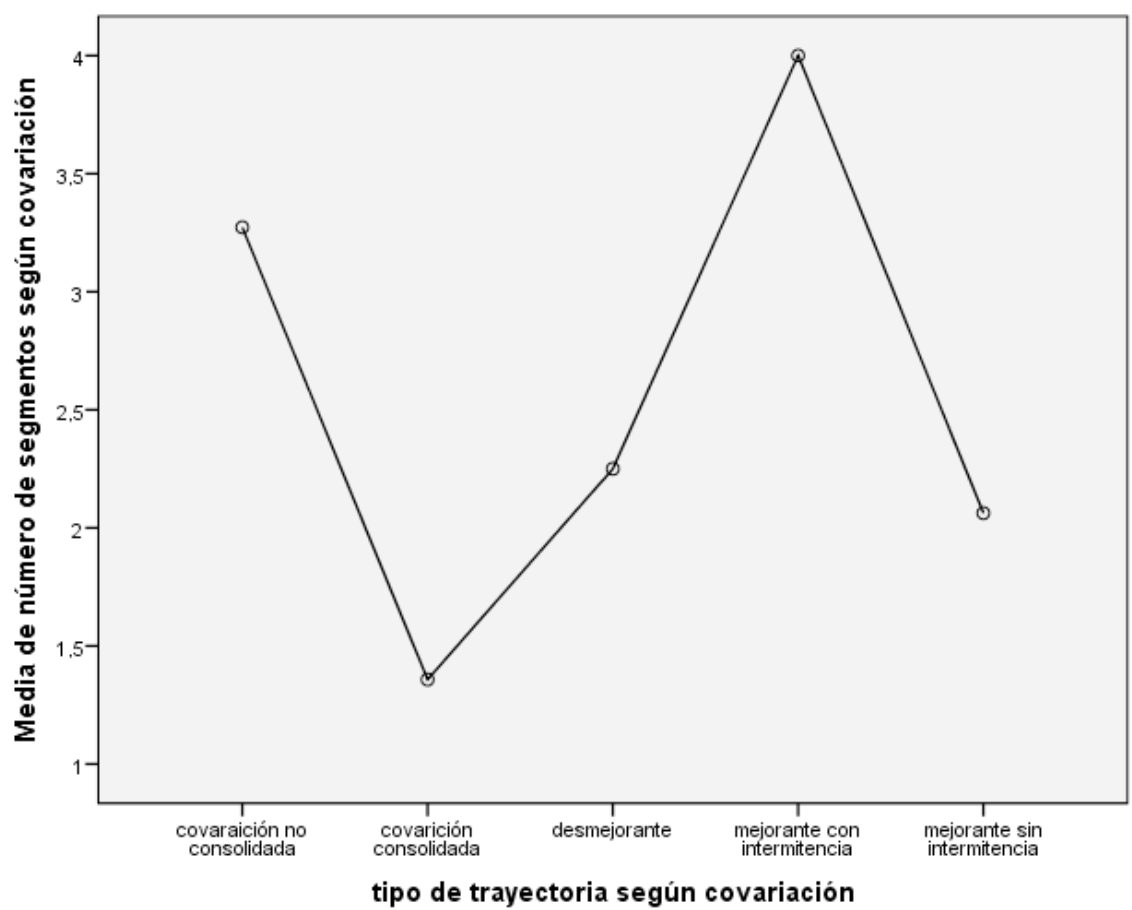

Figura 5. Promedio segmentos según covariación

Fuente: elaboración propia 


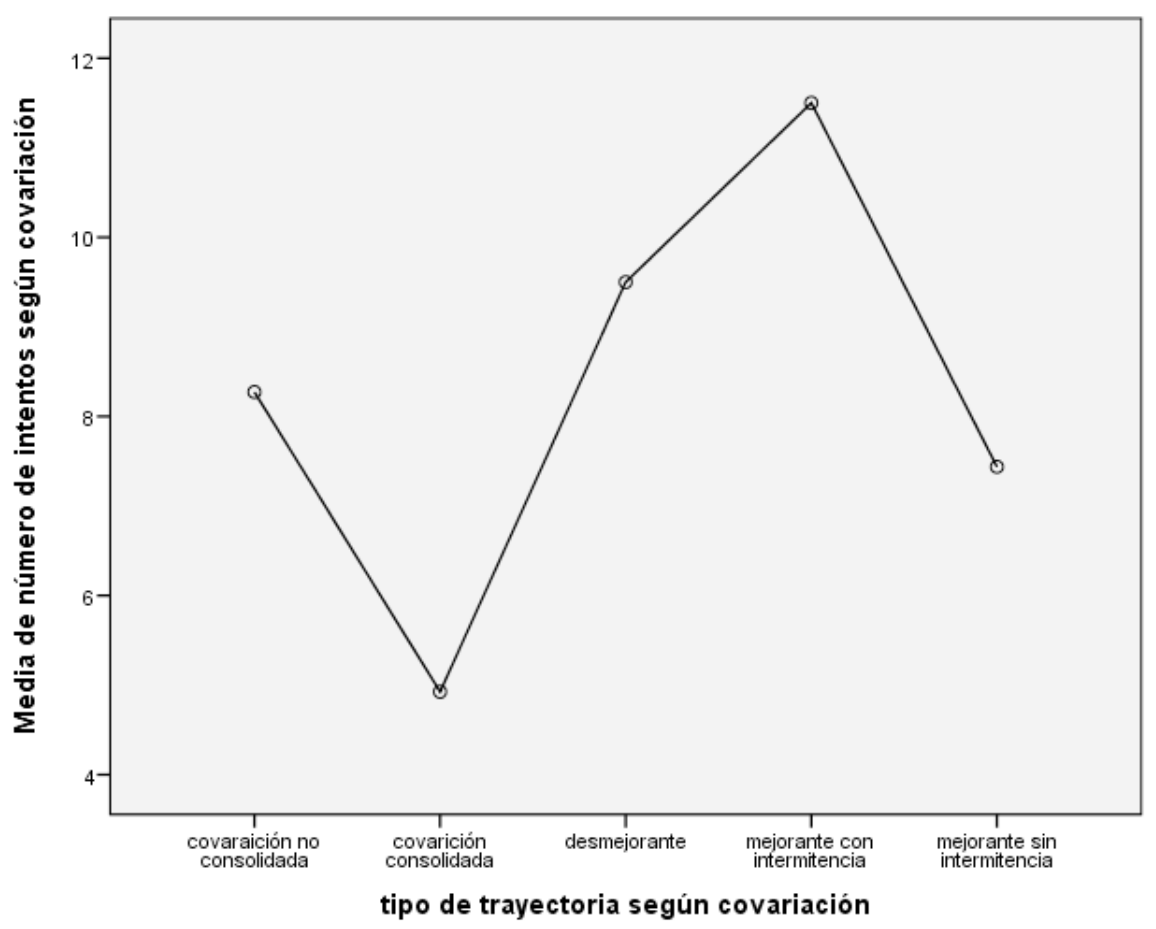

Figura 6. Número de intentos por trayectoria según covariación

Fuente: elaboración propia

dimensión control de variables; la actuación adopta una tendencia más acotada de la covariación que traduce probablemente una intuición de la solución adecuada que conduce al éxito.

En la Figura 6 se observa el lugar de la covariación consolidada respecto de la no consolidada y la desmejorante, respectivamente; en este contexto, los sujetos con covariación consolidada exhiben un menor promedio en el número de intentos, que se traduce en poca variabilidad del tipo de covariación. De igual manera, se destaca que los grupos desmejorante y mejorante con intermitencia difieren de los demás en el promedio de intentos al interior de las trayectorias, lo que se traduce en diferencias significativas respecto de la eficiencia frente a la solución de la tarea.

En síntesis, en esta muestra estudiada podría afirmarse la validez de la hipótesis en gran medida. Las trayectorias de control o covariación consolidada o mejorante sin intermitencia son significativamente más homogéneas y más eficientes que las trayectorias de control o covariación no consolidada o mejorantes con intermitencia. No se pueden afirmar diferencias significativas en el promedio de intentos por segmento, es decir, en la rapidez con la que se produce el cambio.

A continuación, las Figuras 2 a 6 muestran gráficamente los trayectos y trayectorias. Estos mapas permiten visualizar la diversidad de trayectorias que siguen los sujetos frente a la tarea-problema. El primer número del paréntesis indica la cantidad de sujetos que terminan su trayectoria en ese trayecto y el segundo la cantidad de sujetos que continúan más allá.

La Figura 7 destaca cuántos sujetos iniciaron sin control (a), 4 de ellos permanecieron en un procedimiento que no cambió hacia formas de control y 72 continuaron en trayectorias alternas al utilizar diferentes tipos de control de variables. Se observa una trayectoria no consolidada (abababa) y mejorante con intermitencia (ababace).

La Figura 8 describe a los sujetos que iniciaron con los tipos de control b y $\mathbf{c}$ respectivamente (control simple y control simple con más de dos intentos). Se evidencia un cambio en la utilización de las formas de control y trayectorias mejorantes 


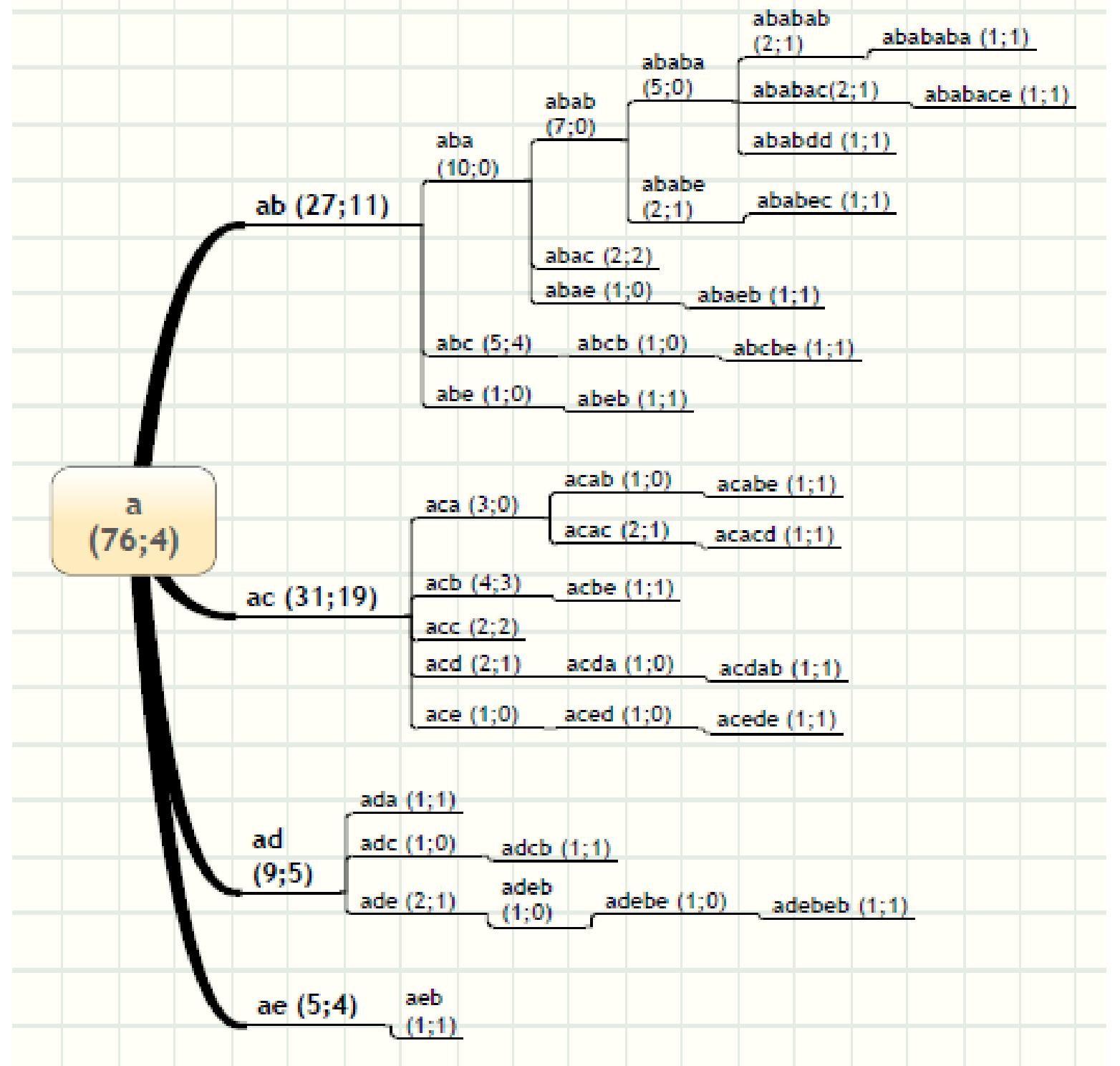

Figura 7. Mapa de trayectos y trayectorias según control y que inician con " $a$ "

Fuente: elaboración propia

que superan la utilización del tipo de control con que iniciaron [(be) y (ccd)].

La figura 9 evidencia el uso inicial del tipo de control cambio con alternancia (d) y cambio controlado (e) al igual que trayectorias mejorantes [(de) y (ede), respectivamente.

La Figura 10 muestra el inicio sin una forma de covariación que en todos los casos configura trayectorias mejorantes.
La Figura 11 expresa tanto trayectorias mejorantes con intermitencia (gifi) y sin ella (gh), que develan igualmente el cambio cognitivo.

Los mapas de las trayectorias según la dimensión covariación comparados con los mapas según control presentan menor diversidad. El contraste entre las trayectorias que inician con no covariación con las que inician con covariación (inician con $\mathbf{g}, \mathbf{h} \mathrm{o}$ i) no es tan fuerte como sí lo es entre las que inician con 


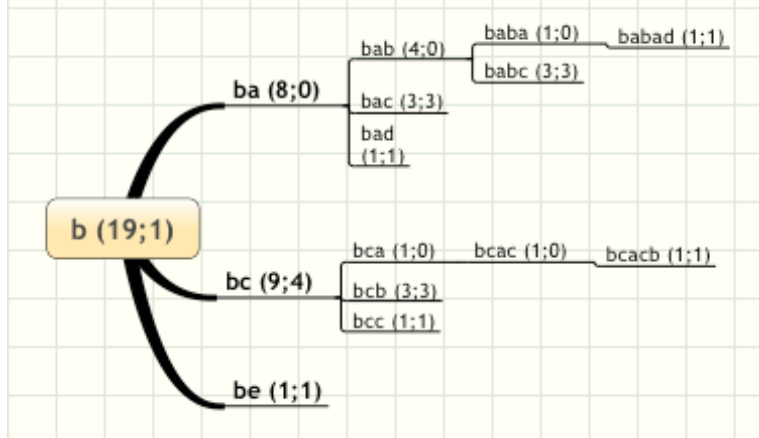

Figura $8 a$

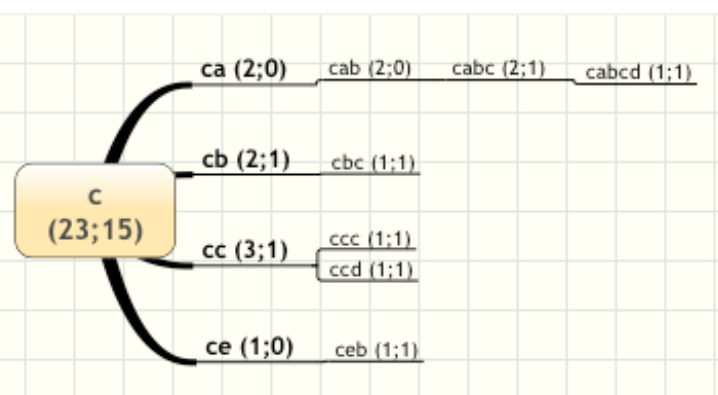

Figura $8 b$

Mapa de trayectos y trayectorias según control y que inician con " $b$ " y "c"

Fuente: elaboración propia

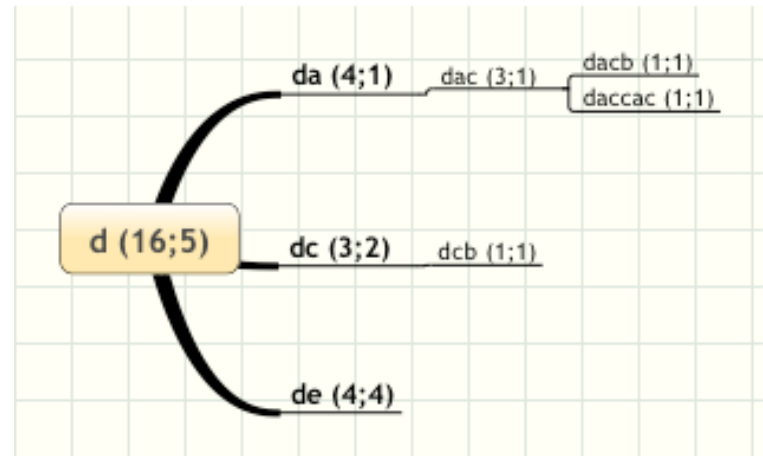

Figura $9 a$

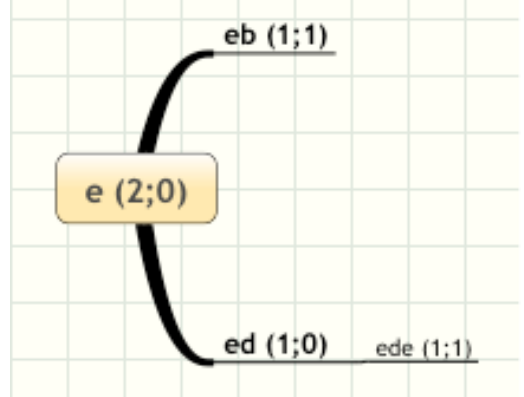

Figura $9 b$

Mapa de trayectos y trayectorias según control y que inician con " $d$ " $y$ " $e$ "

Fuente: elaboración propia

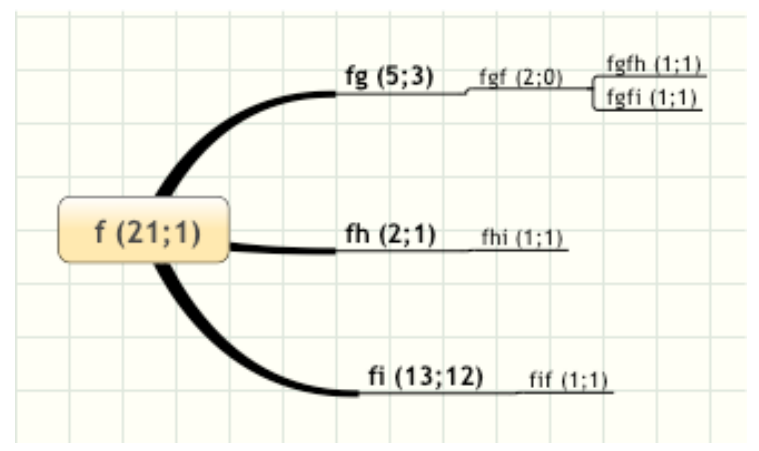

Figura 10. Mapa de trayectos y trayectorias según covariación y que inician con "f"

Fuente: elaboración propia

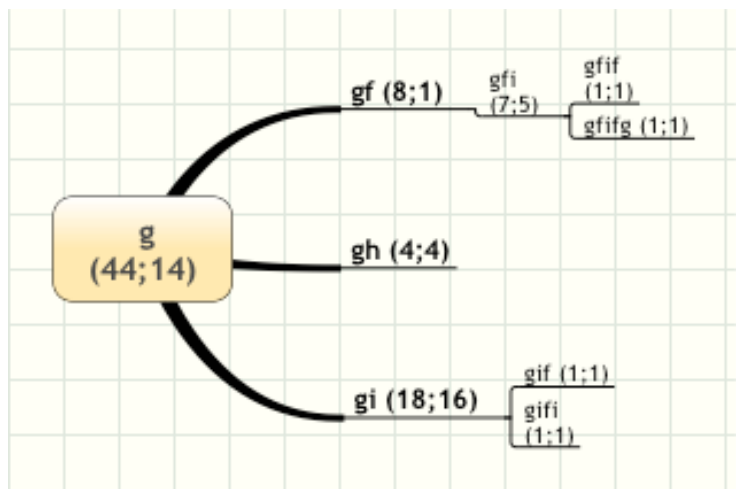

Figura 11. Mapa de trayectos y trayectorias según covariación y que inician con " $\mathrm{g}$ "

Fuente: elaboración propia 


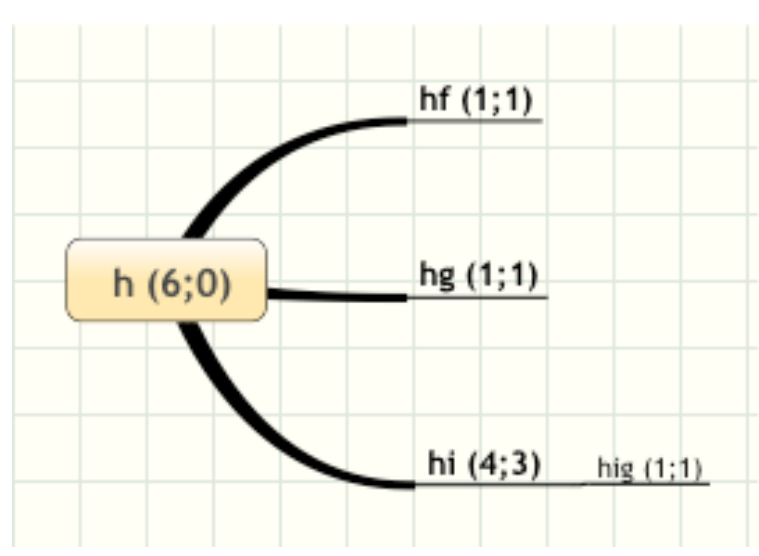

Figura 12a.

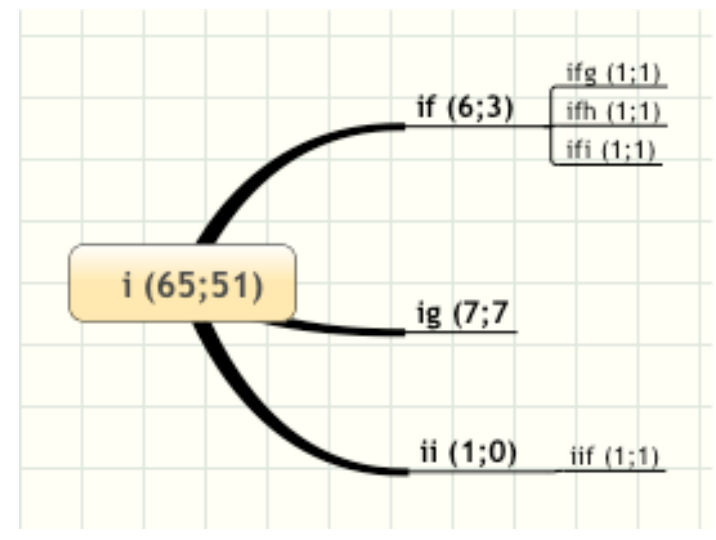

Figura $12 b$.

Mapa de trayectos y trayectorias según covariación y que inician con " $h$ " e " $i$ "

Fuente: elaboración propia

no control y control. Quizá esto se debe a que en esta población los sujetos exhiben desde el comienzo mayor manejo de la covariación, únicamente 21 de los 136 sujetos inician con no covariación, mientras que 76 inician con no control.

Los seis mapas muestran algunos cambios que se producen de forma más frecuente que otros. Según la dimensión control, en esta muestra, en las trayectorias que inician con "a" se da con mayor frecuencia un cambio de $\mathbf{a}=>\mathbf{b}$ o $\mathbf{a}=>$ c que de $\mathbf{a}=>\mathrm{d}$ o que de $\mathbf{a}=>\mathrm{e}$, mientras que según la dimensión covariación las trayectorias que inician sin covariación con mayor frecuencia pasan a covariación mixta $(\mathbf{f}=>\mathbf{i})$ y esto, seguramente, porque con el primer segmento pasan al otro lado de la meta, por lo que el resolutor da valores menores a $\mathrm{V}$ o $\mathrm{T}$ para disminuir $\mathrm{D}$ y logra alcanzar la meta.

El control tipo "b" muestra más inestabilidad que los otros tipos de control. De las 27 trayectorias que cambian $\mathbf{a}=>$ b y que no terminan en este trayecto (Figura 1), 10 tienen un segundo cambio $b=>$ a y

TABLA 5.

$\chi^{2}$ chi-cuadrado covariación por control

\begin{tabular}{lccc}
\hline \multicolumn{1}{r}{ COVARIACIÓN } & Clto(3) & CONTROL & Bedio(2) \\
\hline Alto & 96 & 0 & 66.7 \\
Medio & 4 & 100 & 0 \\
Bajo & 0 & 0 & 33.3 \\
Total & 100 & 100 & 100 \\
$\mathrm{n}$ & 101 & 1 & 3 \\
Chi cuadrado & 54.545 & & \\
$\mathrm{df}$ & 4 & & \\
$\mathrm{P}$ & 0 & & \\
Ccont & 0.585 & & \\
\hline
\end{tabular}

H.: estadística.: $\chi^{2}=0$ Es decir, no existe relación entre covariación y control.

H.: alternativa $\chi^{2}>0$ Es decir, si existe relación entre covariación y control.

Valor crítico 18,47

$\chi^{2}=54.545>18.47 \mathrm{p}<0$.

Fuente: elaboración propia 
algunas de estas vuelven a $\mathbf{b}$ u otro tipo de control y después nuevamente a no control antes de terminar la tarea. Aunque las trayectorias con el trayecto ac después muestran un segundo cambio a no control $(\mathbf{a}=>\mathbf{c}=>\mathbf{a})$, éste es menos frecuente. De las 19 trayectorias que tienen el trayecto ac y que no terminan ahí, sólo 4 regresan a no control (3 del trayecto aca y 1 con el trayecto acda). De las 9 trayectorias con trayecto ad sólo 1 presenta el cambio $\mathrm{d}=>$ a. Las 5 trayectorias de trayectos ae no presentan cambios $\mathbf{b}$ $=>\mathbf{a}$. Quizá estos datos sugieren una posible menor complejidad de control tipo b que de los otros y que podría aparecer en trayectorias sin control con mayor frecuencia que los otros tipos de control, pero los datos de esta muestra no son suficientes para hacer alguna afirmación al respecto.

Los resultados igualmente han sugerido relaciones precursoras entre las dimensiones del control de variables y la covariación, por lo que se realizó una prueba de $\chi^{2}$ chi-cuadrado entre dichas dimensiones. El resultado se expresa en la Tabla 5.

De acuerdo con el coeficiente de contingencia (0.585) no se supone la hipótesis estadística, por lo que se acepta plenamente la hipótesis alternativa de relación. Tanto el chi-cuadrado como el coeficiente de contingencia revelan una relación positiva y significativa entre el control y la covariación. Lo anterior permite plantear que el control podría ser precursor de la covariación.

\section{Discusión}

Puche-Navarro (2008, citado por Vasco \& Henao, 2008) y van Geert y Steebeek (2005) expresan que el desarrollo constituye una trayectoria cambiante, variable, sin una meta fija de antemano a la cual llegar, alejándose de los modelos finalistas. En este sentido, las estadializaciones clásicas si bien evidenciaron el carácter mejorante del desarrollo cognitivo, no lo alcanzaban a describir y explicar, en éste caso, en términos de su verdadero dinamismo variable.

Claramente en esta población es notoria la diferencia en el manejo inicial que exhiben los sujetos para estas dos dimensiones, el control de variables y la covariación: mientras una pequeña parte de la muestra inicia y termina controlando alguna de las variables al ejecutar la tarea, en una gran parte de esta muestra los sujetos exhiben que desde el comienzo están en capacidad de establecer alguna relación entre velocidad $(\mathrm{V})$ y distancia recorrida (D) o entre tiempo (T) y distancia recorrida. Overton (1977) así lo señalaba en términos del cambio variacional que expresa las modificaciones en el nivel del funcionamiento procedimental.

Esta relación de covariación quizá sea únicamente de tipo aditivo (a mayor velocidad, mayor distancia recorrida y a mayor tiempo, mayor distancia recorrida), aunque en algunos casos podría ser de tipo multiplicativo (a $k$ veces $V$ o $k$ veces $T$ corresponde $k$ veces D), pero en este estudio, por la información obtenida, no se puede identificar con fiabilidad cuándo se trata de una covariación aditiva o de una multiplicativa.

Podría decirse que estos cambios drásticos de control a no control o de covariación a no covariación, o viceversa, se presentan en sujetos que buscan mejorar su estrategia para resolver la tarea y están en condiciones de intentar estrategias mejores, mientras que hay sujetos que no mejoran su estrategia, bien porque no la buscan o porque buscándola no logran encontrarla. Lo anterior se inscribe en los planteamientos teóricos de Pozo (2009) acerca del desarrollo cognitivo, al distinguir tres tipos de transformaciones, como enriquecimiento, ajuste de las estructuras cognitivas y reestructuración de las mismas. En el contexto de la presente investigación se evidencian el cambio y la transformación como ajustes frente a la utilización de las dimensiones del control de variables y de la covariación.

Aquellas trayectorias que indican mayor inestabilidad en el control son las que exhiben mayor frecuencia de cambio (menor valor de número de intentos/segmento), mientras que aquellas que indican más estabilidad presentan cambios con menos frecuencia (mayor valor de número de intentos/ segmento). Esta estabilidad para el cambio puede darse en dos casos extremos: a) bien porque el sujeto muestra que no maneja control (o covariación) al iniciar la resolución de la tarea y continúa así a lo largo de la resolución de la misma ob) por el contrario, porque ya desde el comienzo manejaba el control (o la covariación) y lo mantiene durante la resolución. Siegler (1996) y Farrington, Vanuxemy Stiller 
(2009), explicarían lo anterior como la superposición de estrategias, formas y tipos de actividad cognitiva según las cuales los sujetos, si bien poseen variadas formas frente a la solución de los problemas, eligen la que más se adecúa a la naturaleza del mismo.

El mayor o menor manejo de las dos dimensiones, control y covariación, tiene incidencia directa en la mayor o menor eficiencia con la que se resuelve la tarea. A mayor manejo de control o a mayor manejo de covariación las trayectorias tienen menos intentos.

Los datos muestran una clara diferencia en la eficiencia con la que se resuelve la tarea cuando no se manejan ambas dimensiones, que cuando sí se manejan. Sin embargo, el hecho de tener una única trayectoria de no control y no covariación da poca validez a esta afirmación, de ahí que sea necesario acopiar mayor información de otras muestras en términos de una mayor densidad para cada categoría.

Los datos sugieren una posible menor estabilidad del control tipo $\mathbf{b}$ que de los otros tipos y que podría interpretarse como que este tipo de control es de menor complejidad que los otros, porque podría aparecer en trayectorias sin control con mayor frecuencia que los otros tipos de control, aunque los datos de esta muestra no son suficientes para hacer alguna afirmación contundente al respecto.

El estudio realizado permitió identificar una alta variedad de trayectos y trayectorias determinadas por cada una de las dimensiones (control y covariación). La presencia intra e inter de variedad de trayectorias indica la variabilidad del cambio cognitivo y la tendencia mayoritaria de rutas mejorantes muestra el carácter de progreso del cambio cognitivo en la solución de este problema. Las trayectorias con manejo de la dimensión covariación (independientemente de que tengan control o no) resultan más eficientes que aquellas en las que hay control pero no hay covariación.

En esta línea de análisis surgen las siguientes preguntas: ipodría afirmarse que la dimensión control, en tanto estrategia de resolución de la tarea, comparada con la dimensión covariación, en tanto compresión de la tarea, es menos determinante en la resolución eficiente de la misma?, ison las dos dimensiones, control de variables y covariación, constructos precursores en ese orden uno del otro y además adecuados para caracterizar el cambio como novedad cognitiva? icuáles son las representaciones mentales que soportan los patrones de ejecución?

Esta indagación sugiere continuar una línea de investigación que requiere nuevas miradas y perspectivas teóricas y metodológicas. Ossa (2011) propone analizar la variabilidad a partir de regresiones utilizadas en los modelos de crecimiento logísticos, los cuales permiten dar cuenta del cambio en tanto están basados en diferentes modelos (modelo de Malthus, modelo de Verhulst, modelo de Lotka y Volterra: modelo de la relación depredador-presa) y fórmulas planteadas desde el cálculo diferencial: método matemático que permite estudiar la variación y el ritmo en el cual varían las funciones.

En el epílogo de la presente indagación, los autores se adhieren a una perspectiva del cambio evolutivo epigenético y probabilístico de los procesos psicológicos, desde el nacimiento hasta la muerte, como explosión de estados y procesos del desarrollo bajo condiciones de arranque dados por ser miembros de la especie homo sapiens-sapiens, propia de los humanos modernos, soportados por un mecanismo de búsqueda y establecimiento de nuevas elecciones y relaciones entre elementos marcados por puntos de inflexión, al estilo de las redes sociales, en las que existen elementos invitados, aceptados, en espera o excluidos de forma inevitable. Para el sujeto en desarrollo siempre estarán más o menos explícitos los diversos caminos y los pequeños espacios nunca elegidos, pero sí soñados y deseados.

\section{Referencias}

Bermejo, V. (2005). Microgénesis y cambio cognitivo: Adquisición del cardinal numérico. Psicothema, 17(4), 559-562.

Escobar, H. (2003). Historia y naturaleza de la psicología del desarrollo. Universitas Psychologica 2(1), 71-88.

Farrington, L., Vanuxem, S., \& Stiller, J. (2009). Patterns of problem-solving in children's literacy and arithmetic. British Journal of Developmental Psychology, 27, 815-834. http://dx.doi. org/10.1348/026151008x383148

Gutiérrez, F., \& Carriedo, N. (2002). Métodos en el estudio del desarrollo cognitivo. En F. Gutiérrez, J. A. García, \& N. Carriedo (Eds.), Psicología Evolutiva 
II: Desarrollo cognitivo y lingüístico Volumen I (pp. 50-85). Madrid: UNED.

Gutiérrez, F., García, J., \& Luque, J. L. (2002). Los enfoque dinámicos. El conexionismo y los sistemas dinámicos. En F. Gutiérrez, J. A. García, \& N. Carriedo (Eds.), Psicología Evolutiva II: Desarrollo cognitivo y lingüístico Volumen I (pp. 148-177). Madrid: UNED.

Karmiloff-Smith, A. (1994). Más allá de la modularidad. La ciencia cognitiva desde la perspectiva del desarrollo. Madrid: Alianza Editorial.

Montealagre, R. (2011). La solución de problemas cognitivos en estudiantes de psicología. Acta Colombiana de Psicología, 14(1), 119-138.

Navarro-Roldán, C. (2008). Comprensión de sistemas de engranajes: un estudio del cambio cognitivo y las herramientas cognitivas en niños de cuatro años [Spanish]. Universitas Psychologica, 7(2), 411-424.

Ossa, J. (2011). Funcionamiento cognitivo: Un inextricable juego de pérdidas y ganancias. Acta Colombiana de Psicología, 14(2), 45-55.

Ossa, J. (2013). Matrices de transición y patrones de variabilidad cognitiva. Universitas Psychologica, 12(2), 559-570.

Ossa, J. C., \& Puche-Navarro, R. (2010). Modelos bayesianos y funcionamientos inferenciales complejos. Acta Colombiana de Psicología. 13(2), 119-128.

Overton, W. (1997). Developmental psychology: philosophy, concepts, and methodology Handbook of child psychology: Theoretical models of human development. New York: John Wiley \& Sons.

Piaget J. (1977). Biología y Conocimiento. Madrid, España: Siglo XXI Editores, S.A.

Piaget, J. (1979). El mecanismo del desarrollo mental. Madrid: Editora Nacional.

Pinker, S. (2013). La tabla rasa: la negación moderna de la naturaleza humana. Barcelona: Ediciones Paidós Ibérica

Pozo, J. I., \& Gómez Crespo, M. A. (2009). Aprender y enseñar ciencia. Del conocimiento cotidiano al conocimiento científico. Barcelona: Ediciones Morata.

Pozo, J. I., \& Gómez Crespo, M. A. (2009). Del conocimiento cotidiano al conocimiento científico: más allá del cambio conceptual. En J. I., Pozo, \& M. A. Gómez Crespo, Aprender y enseñar ciencia.
Del conocimiento cotidiano al conocimiento científico. Barcelona: Ediciones Morata.

Puche-Navarro, R. (2008). Érase una vez el desarrollo. En J. Larreamendy-Joerns, R. Puche-Navarro, \& A. Restrepo (Eds.), Claves para pensar el cambio: Ensayos sobre psicología del desarrollo. (pp. 30-68). Bogotá: Universidad de los Andes, Centro de Estudios Socioculturales e Internacionales.

Puche-Navarro, R., Combariza, E., \& Ossa, J. C. (2003). La naturaleza no lineal de los funcionamientos inferenciales: un estudio empírico con base en el humor gráfico. Avances en Psicología Latinoamericana, 30(1), 27-38.

Siegler, R., \& Crowley, K. (1991). The microgenetic method. A direct means for studying cognitive development. American Psychogist, 46(6), 606-620. Recuperado el 27de Marzo de 2015 de http://dx.doi. org/10.1037/0003-066X.46.6.606

Siegler, R. (1996). Emerging Minds: The process of Change in Children's Thinking. Oxford: Oxford University Press.

Torbeyns, J., Arnaud, L., Lemaire, P., \& Verschaffel, L. (2004). Cognitive change as strategy change. En A. Demetriou, \& A. Raftopoulos (Eds.) Cognitive Developmental Change: Theories, Models and Measurement. Cambridge University Press.

van Geert, P., \& Steenbeek, H. (2005). A complexity and dynamic systems approach to development: Measurement, modeling and research. In K. W. Fischer, A. Battro, \& P. Lena (Eds.), Mind, Brain and Education. Cambridge: Cambridge University Press.

van Geert, P., \& van Dijk (2002). Focus on variability: New tools to study intra-individual variability in developmental data. Infant Behavior $\mathcal{E}$ Developmental 25, 340-374.

Vasco, C. E., \& Henao, G. C. (2008). Elementos y Modelos del Desarrollo: Una revisión del Concepto. En J. Larreamendy-Joerns, R. Puche-Navarro, \& A. Restrepo (Eds.), Claves para pensar el cambio: Ensayos sobre psicología del desarrollo (pp. 1-27). Bogotá: Universidad de los Andes, Centro de Estudios Socioculturales e Internacionales.

Yan, Z., \& Fischer, K. (2002). Always under construction: Dynamic variations in adult cognitive microdevelopment. Human Developmental processes, 2(2), 39-62. 
